\title{
A Case of Nicotine vs. Psychosis
}

\author{
Kabuye A*, Mokhtar M and Emmanuel RO \\ Bon Secours Hospital, Baltimore, Maryland, USA \\ *Correspondling author: Kabuye A, Bon Secours \\ Hospital, Baltimore, Maryland, USA
}

Received: July 12, 2018; Accepted: August 17, 2018;

Published: August 24, 2018

\begin{abstract}
Ekbom's Syndrome, also known as delusional parasites, is a psychotic disorder in which a patient is convinced that they are infested with some sort of parasite. There is usually a triggering event in most cases, but not always identified. In this case, we report a diagnosis of Ekbom's syndrome on a female patient who believes that parasites have infected her body and now are everywhere.
\end{abstract}

Keywords: Ekbom's Syndrome; Psychosis; Psychotic disorder

\section{Case Presentation}

A 59-year old female patient was admitted to the psychiatric ward for delusions of bug infestation all over her body and everywhere she looks. She states that these bugs first attacked her face, in June 2017 while she was in the basement and they are now all top of the refrigerator and her stove. She states that she has killed some and also has bitten some of them as they have made it to her mouth. The patient said every time she smoked a cigarette she would see eggs of the bugs falling out of her skin. She further states that she lost weight as she tried different remedies like drinking honey and other substances to try and kill the parasites. She describes the parasites as oval-black shaped with a pointy spine that they use to burrow through her skin. She further reports that these parasites do not move anywhere but she can feel them burrowing through her skin and causes her pain. The patient also stated that the first time the parasites attacked her, they caused her to scratch her face several times. The severe scratching on her face left scars, which the patient attributes as marks which by the parasites recognize. With this mark, the bugs will attack her and not anyone else in the family. There was no evidence of parasites or any bugs on her. She stated that her symptoms were alleviated with cigarette smoking.

The patient was admitted after a referral from the dermatologist who stated that the excessive scratching the patient was experiencing was not due to a skin condition. Upon admission, the patient stated that her body and her home were infested with bugs and she continues to state that these bugs were everywhere as well as living inside her while laying eggs. The patient further reports that she sees them entering her skin and she tries to scratch them out. She points out the several scars on her body and stating the bugs entered the skin here. She stated that she has captured some of them and showed it to her sister; when the sister was asked about this, she said she had collected dirt in a container and that no bugs were present inside.

\section{Past medical history}

Patient has past medical history of HTN, asthma, anxiety, opiate abuse Physical Exam.

General: The patient is oriented to place, time and person, appears well nourished and age appropriate.

Heent: head showed several excoriations on the face of varying healing stages, hyperpigmentation patches on the faces and papules on the nose, eyes were non-icteric, EOM intact and PERRL, TMs translucent and mobile with hearing intact, no mucosal lesions in mouth with moist mucous membranes, neck was supple with some excoriations of varying healing stages.

Heart: Regular rate and rhythm no murmurs or gallop.

Lungs: Clear to auscultation and percussion, no rhonchi rales or wheezing. GI: normal bowel sounds with no guarding or tenderness to palpation.

Neuro: CN 2-12 intact, with no focal deficits.

MSK: Normal gait and station, reflexes normal, strength equal in all extremities.

Behavior: Patient though is very defensive about her delusion.

Speech: Fast-paced slurred speech.

Mood: Depressed.

Affect: Flat.

Thought process: Circumstantial.

Thought content: Thoughts were fixed on parasites, stopped her from sleeping due to a fear of parasites entering her mouth and continual attacks.

Insight and judgment: Insight was poor and judgment was fair.

Memory: Intact.

Intellectual function: Intact.

Attention: Distracted.

Suicidality and homicidality: No SI or HI.

\section{Labs}

WBC- 4.45, RBC - 3.0, Hemoglobin 10.8, hematocrit 33.9, calcium 8.7, alkaline phosphate 125, AST of 33, ALT of 24, Urinalysis was unremarkable. Urine toxicology positive for benzodiazepines, cocaine, codeine, morphine, and methadone.

\section{Hospital course}

The patient was hospitalized after the scars were determined to not be of a dermatological origin. During her stay, she had one episode of psychotic agitation and was chemically restrained. Upon becoming 
responsive the patient remained delusional about the parasitosis inside her body and was started on Geodon, Depakote, and Cogentin. During her hospital stay, she was never allowed to smoke.

\section{Discussion}

Nicotine is known to affect several neurotransmitters largely because of the similarity or increase productions of some neurotransmitters. Take for example nicotine and acetylcholine molecule is similar [1]. This nicotine property could be useful in patients with Alzheimer's or huntington, but this will not be discussed further as it is beyond the scope of the paper. Nicotine is known to increase the release of dopamine. This can prove to be useful in schizophrenic patients who experience negative symptoms like, avolition, anhedonia, asociality, alogia because these symptoms present with a decrease in dopamine in the Prefrontal Cortex. Administering nicotine would upregulate the release of dopamine and Most of these receptors in the Central Nervous System for nicotine are distinguishable by subunits they have and also different thresholds they have for nicotine.

Nicotine has been shown to have an effect primarily on dopamine, glutamate, GABA, serotonin, and norepinephrine [1,2]. An imbalance of these neurotransmitters can present with a wide array of symptoms like depressions and anxiety which can then lead to pyschosis. Depressed patients are known to have low levels of norepinephrine and serotonin, while patient with anxiety are known to have low levels of GABA and serotonin. Nicotine is known to stimulate the release of various neurotransmitters. Although continued use of nicotine can present addiction problems but the nicotine molecule can be manipulated to remove its addictive effect while maintaining its ability to increase levels of the various brain chemicals that can alter mood [3].

Evidence has also shown that smoking may reduce some side effects of neuroleptic medications to cause Parkinsonism [4]. Administration of nicotine patches has shown a reduction in Akathisia, a common side effect of many antipsychotics [5-12].

There is evidence of the fact that nicotine has several interactions with various receptors and neurotransmitters in our bodies. With this in mind, we can further explore the benefits of nicotine and have them as alternatives patients who seem to have compliance issues due to adverse effects of schizophrenia medication.

\section{Conclusion}

The science behind the ill effects of smoking is well documented and abundantly clear, and by no means should this paper be used as an argument to start smoking. However, when it comes to the problems faced in the psychiatric community of helping patients, risk versus benefits of nicotine may give interesting insight.

As mentioned earlier, one major problem faced by the psychiatric community, other than the symptoms themselves, is that of side effects. There is some interesting research that shows that side effects can be curbed with the help of nicotine, as well as overall help with the psychosis as in the case of the patient in question. Too many of the patients in the wards have something to the tune of "illicit drug use" in their files, which can always be interpreted as drug use which can bring on mental illness, but the possibility that these patients are merely trying to self-medicate should not be ruled out.

What prompted the writing of this case report about this patient is that one of the alleviating symptoms for her was having a smoke. Once on the psych ward, no one is allowed to leave unless discharged, meaning that not once was she allowed access to her alleviating symptom. That goes without saying, just because a patient states that they feel better jumping off of a roof, we are not obligated to help them find the highest building. That being said, this isn't the first time that a patient has stated that smoking helped with their illness and perhaps the stigma of cigarette smoke should be put aside temporarily to see if perhaps this is an avenue worth exploring. After all, if Parkinsonism or tardive dyskinesia (TD) are potential side effects worth tolerating for a potential relief, why then, should the same consideration not be given to nicotine use? Considering that Parkinsonism can be caused overnight if dopaminergic drugs are put on too quickly, or the fact that TD is irreversible, the medications are still recommended because the benefits outweigh the risks. With nicotine, the obvious risk is smoking and this complicates the risk $v s$. benefits considerably. However, if there is some way to either administer the nicotine via a patch or give the nicotine in conjunction with current therapy for the cases that do not seem to be responding to typical medications and to get similar results which patients say they receive from smoking then these methods should be considered on a benefit $v$ s. side effects. There are far too many unanswered questions surrounding this topic, and a great deal of it is due to the stigma surrounding nicotine

Psychiatric cases are some of the most difficult to treat, greatly due to our lack of knowledge of what really is going wrong in the brain. Pharmacological treatments and behavioral therapy are among the best ways to treat the majority of patients; however, there are some that only get "improved" written on their action and plan because no further treatments options are available. The unfortunate truth is that there is too much stigma behind illicit drugs and tobacco to be taken seriously as treatment options, but this is not a good reason to avoid further studies on their efficacy with respect to mental illness.

We thank Bon Secours for the opportunity to help and further the boundaries of medical science.

\section{References}

1. Gray JA, Mitchell SN, Joseph MH, Grigoryan GA, Dawe S, Hodges H. Neurochemical mechanisms mediating the behavioural and cognitive effects of nicotine. Drug Dev Res. 1994; 31: 3-17.

2. Levin ED, Simon BB. Nicotinic acetylcholine involvement in cognitive function in animals. Psychopharmacology. 1998; 138: 217-230.

3. Duke University Medical Center. Nicotine Lessens Symptoms Of Depression In Nonsmokers. Science Daily. 2006.

4. Goff DC, Henderson DC, Amico E. Cigarette smoking in schizophrenia: relationship to psychopathology and medication side effects. Am J Psychiatry. 1992; 149: 1189-1194.

5. Anfang MK, Pope HG Jr. Treatment of neuroleptic-induced akathisia with nicotine patches. Psychopharmacology. 1997; 134: 153-156.

6. Yang YK, Nelson L, Kamaraju L, Wilson W, McEvoy JP. Nicotine decreases bradykinesia-rigidity in haloperidol-treated patients with schizophrenia. Neuropsychopharmacology. 2002; 27: 684-686.

7. Chouinard G, Arnott W. Clinical review of risperidone. Can J Psychiatry. 1993; 38: S89-S95.

8. Nyback H, Nordberg A, Langstrom B, Halldin C, Hartvig P, Ahlin A, et al. 
Attempts to visualize nicotinic receptors in the brain of monkey and man by positron emission tomography. Prog Brain Res. 1989; 79: 313-319.

9. Olincy A, Young DA, Freedman R. Increased levels of the nicotine metabolite cotinine in schizophrenic smokers compared to other smokers. Biol Psychiatry. 1997; 42: 1-5.

10. Smith RC, Singh A, Infante M, Khandat A, Kloos A. Effects of cigarette smoking and nicotine nasal spray on psychiatric symptoms and cognition in schizophrenia. Neuropsychopharmacology. 2002; 27: 479-497.
11. Stein EA, Pankiewicz J, Harsch HH, Cho JK, Fuller SA, Hoffmann RG, et al. Nicotine-induced limbic cortical activation in the human brain: a functional MRI study. Am J Psychiatry. 1998; 155: 1009-1015.

12. Uck A, Polat A, Bozkurt $\mathrm{O}$, Meteris $\mathrm{H}$. Cigarette smoking among patients with schizophrenia and bipolar disorders. Psychiat Clin Neurosci. 2004; 58 434-437
Austin J Clin Case Rep - Volume 5 Issue 2 - 2018

ISSN : 2381-912X | www.austinpublishinggroup.com

Kabuye et al. (C) All rights are reserved
Citation: Kabuye A, Mokhtar M and Emmanuel RO. A Case of Nicotine vs. Psychosis. Austin J Clin Case Rep. 2018; 5(2): 1134 